\title{
CO-DESNET: AN APPROACH TO MODELING COLLABORATIVE DEMAND AND SUPPLY NETWORK
}

\author{
Agostino Villa and Irene Cassarino \\ Dipartimento di Sistemi di Produzione ed Economia dell'Azienda \\ Politecnico di Torino, c.so Duca degli Abruzzi, 24, 10129 - Torino (Italy) \\ Phone: +39-011-564.7233; fax: -564.7299; e-mail: agostino.villa@polito.it
}

\begin{abstract}
Present evolution of multi-firm supply chains and industrial districts urges to have at disposal procedures and methods for the organization and management of a COllaborative DEmand \& Supply NETwork (CO-DESNET. The crucial point in organizing and managing such networks is "to assure good collaboration among partners": interactions among the firms as well as the connecting information pattern have to be designed accordingly. The presentation of a new model of a CO-DESNET is the scope of the paper: based on this model, conditions verifying how collaborative operations of the firms included in the network will occur, are derived.
\end{abstract}

\section{INTRODUCTION}

In several European countries, groups of Small-Mid scale Enterprises (SMEs) aim to cooperate together, forced by the globalization of markets. The result of these agreements usually is a Demand \& Supply Network (DESNET), which is structured as an "extended virtual enterprise", i.e. a temporary network of several firms which decide to cooperate together in a common given value chain for a limited time horizon.

A special interest of the industrial bodies is reserved to networks of firms characterized by "co-operation agreements" signed for a finite time horizon, and stated such that each firm could partially interact with the other network partners (i.e. each firm could also have a proper market share, thus involving in the network only a part of their own core business). The resulting new DESNET can have a finite life and it does not completely reduce the autonomy of any component firm, because each one can still produce items for proper clients, and then operate in a proper market segment (Villa, 2001). More precisely, all the enterprises which agree to be included into a $D E S N E T$ and then be active inside the same supply chain, must sign an agreement to co-operating together in defining common production plans for specific products.

The design, organization and management of a "temporary DESNET" requires to apply new models of the network operations, based on the concepts of the multi-agent organizations (Zheng \& Possel-Dolken, 2002) and defined in formal terms according to the theory of large-scale dynamic systems optimisation (see Sethi \& Zhang, 1994).

Depending on above mentioned requirements, a DESNET will be here formally 
stated as a virtually connected chain of service stages, each one containing either a firm or a set of parallel firms, each firm with its proper autonomous decision-maker, denoted "agent" (Huang and Nof, 2000, Farantin et al, 1998, Yung \& Yang, 1999): each agent aims to cooperate with the others, but also wants to obtain the best profits for his own enterprise. Each service stage is connected to the upstream stages and to the downstream ones through a virtual market place (Wellman, 1993): it means that each firm will negotiate contracts for producing goods with downstream (buyer) firms as well as contracts for acquiring materials with upstream (supplier) firms. This negotiation opportunity is a qualifying character of a DESNET. Since each firm aims to gain its own best income, it utilizes the DESNET to which it belongs as a frame within which a "good negotiation" can be performed. Here "good negotiation" means that an agreement between each pair of "consecutive agents" (belonging to two consecutive stages of the chain) can be found such as to satisfy both agents, because both aim to being cooperative but, at the same time, want to make profits: then the desired agreement should assure a sufficient income to both of them.

The concepts above introduced allow detailing the real problem now facing supply chain designers and organizers (Villa, 2002): under which conditions does an individual enterprise find convenient to sign a temporary agreement for becoming a partner within a multi-agent DESNET?

Industrial experience suggests that this is a multi-faced problem depending on economic, technological and managerial considerations (Simchi-Levi \& Kaminski, 2000). This paper will approach the problem according to the management point of view. And the goal is to develop a model of the multi-agent management problem in a DESNET such as to analyse how cooperation of partners could be enforced.

The paper contents are as follows. Section 2 introduces a model of the problem of managing interactions among the component firms of a DESNET. Section 3 will discuss the inter-firm negotiation problem, in order to derive conditions which can motivate cooperation among firms. The Section 4 will summarize final considerations about a collaborative management of local autonomous agents.

\section{MODELING THE DESNET MANAGEMENT PROBLEM}

The proposed model of a DESNET is based on the idea that such a networked industrial system consist of an "open commercial system" within which two component firms interact together by exchanging material and financial resources. For each material resource (i.e., parts of a common final family of products), a proper market place exists on which this resource is negotiated through a proper monetary value, between the supplier (i.e., the resource producer) and a customer (i.e. the purchaser who will utilize the resource in its own production sequence).

In a DESNET, considering the complete production cycle of a final product, each buyer will purchase at least a resource to apply his own manufacturing operations in order to transform it into a new item with more added value, to be sold in a downstream market place. So, the principal functions of any component firm are: purchasing, transforming (through either manufacturing or servicing), and selling of items. In 
a Collaborative DESNET (denoted in the following CO-DESNET $T^{\prime}$, as the European project to which the paper is referred), this set of dynamic commercial interactions occurs into a co-ordinated protected industrial network. Partners indeed are connected together through collaboration agreements, which reflects into effectiveness of the commercial negotiations. These agreements should give each component firm a sufficient assurance of economic survival, by suitable management of usual "hard" business relations between large-scale and small-scale firms.

The model presented in the following aims at allowing an easy but correct evaluation of costs and advantages of the above sketched regulated industrial system. To this aim, a CO-DESNET composed by 4 enterprises, belonging to 2 different stages, is considered, together with a final customer. Two market places, one internal and one between producers and the final customer, define the interactions among the agents. At the material input stage, two firms (denoted by index $n=1,2$ ) produce parts to be sold to the two firms belonging to the following stage (denoted by index $n=3,4$ ). These last ones apply final operations to transform parts into products, to be sold to the final customer. The customer's exogenous demand is denoted by $A_{t}$.

Each agent $n$ is modelled by a production optimisation problem: to maximize the firm profit (i.e. the difference between selling return and production costs) with respect to produced volume and price, under the constraints describing the inventory dynamics and the production capacity saturation.

The model for the first stage component firms $n=1,2$ is as follows:

$$
\begin{aligned}
& \min _{X, P, Y} J_{n}=\sum_{t}\left(-P_{n, t} X_{n, t}+c_{n} I_{n, t, F}+r_{n} Y_{n, t}\right) \\
& I_{n, t, F}=I_{n, t-1, F}+Y_{n, t}-X_{n, t} \\
& Y_{n, t} \leq C_{n} \\
& X_{n, t}, I_{n, t, F}, Y_{n, t}, P_{n, t} \geq 0
\end{aligned}
$$

where the following notations have been used, for the first stage component firms $n=1,2$, (all referred to time period $t$ ): $P_{n, t}$ is the price to sell items from supplier $n$ to the second-stage buyers; $X_{n, t}$ is the volume sold from supplier $n ; Y_{n, t}$, the volume produced by supplier $n ; I_{n, t, F}$, the output storage of finished parts at supplier $n ; c_{n}$, the storage unitary cost; $r_{n}$, the production unitary cost, and $C_{n}$, the production capacity of supplier $n$.

Referring to the second stage component firms, $n=3,4$ (denoted as "buyers"), the related models are as follows:

\footnotetext{
${ }^{1}$ CO-DESNET is the acronym of the Coordination Action (CA) project $n^{\circ}$ IST-2002-506673/ Joint Call IST-NMP-1, supported by the European Commission, Information Society Directorate-General, Communication Networks, Security and software, Applications, under the coordination of Politecnico di Torino, Prof. A. Villa, and with EC Official Dr. F. Frederix. This paper refers to research results developed for preparing the CA proposal at Politecnico di Torino.
} 


$$
\begin{aligned}
& \min _{Q, Z, W, D} J_{n}=\sum_{t}\left(Q_{n, t} Z_{n, t}+r_{n} W_{n, t}+c_{n, M} I_{n, t, M}+\right. \\
&\left.+c_{n, P} I_{n, t, P}-S_{t} D_{n, t}\right) \\
& I_{n, t, M}= I_{n, t-1, M}+Z_{n, t}-W_{n, t} \\
& I_{n, t, P}= I_{n, t-1, P}+W_{n, t}-D_{n, t} \\
& W_{n, t} \leq C_{n} \\
& Z_{n, t}, I_{n, t, P}, I_{n, t, M}, W_{n, t}, S_{t} \geq 0
\end{aligned}
$$

with the following notations: $Q_{n, t}$ is price to purchase items by buyer $n$ from the firststage suppliers at the internal market place; $Z_{n, t}$, the volume purchased by buyer $n$; $D_{n, t}$, the volume sold by buyer $n$ to the downstream final client; $A_{t}$, the external demand from final client; $W_{n, t}$, the volume produced by buyer $n ; I_{n, t,}$, the output storage of final products produced by buyer $n ; I_{n, l, M}$, the input storage of parts to be used by buyer $n ; S_{t}$, the unitary price for selling final products during period $t ; c_{n, P}$, the unitary cost of final products storage and $c_{n, M}$ is the unitary cost of input storage of parts.

Since the four enterprises belong to the same CO-DESNET, the network model is completed by introducing some conditions regulating interactions among the partners.

I. Conditions stating that production volumes must be balanced with internal and final demands:

I-a. All parts produced by firms in the upstream stage have to be purchased by firms of the second stage:

$$
\sum_{n=1,2} X_{n, t}=\sum_{n=3,4} Z_{n, t}
$$

I-b. The final client demand has to be fully satisfied:

$$
\sum_{n=3,4} D_{n, t}-A_{t}=0
$$

II. Conditions stating that prices of parts in the internal market place have to be balanced:

$$
P_{1, t}-P_{2, t}=0(12) ; \quad P_{1, t}-Q_{4, t}=0(13) ; \quad Q_{3, t}-Q_{4, t}=0(14) \text {. }
$$

III. Condition stating that the production volumes of the firms belonging to a same stage have to be balanced according to the respective efficiency:

$$
\frac{1}{C_{1}}\left(X_{1, t}\right)^{2}-\frac{1}{C_{2}}\left(X_{2, t}\right)^{2}=0
$$


IV. Condition stating that the volumes of final products sold by the firms belonging to the final stage, to the end customer, have to be balanced according to the respective efficiency:

$$
\frac{1}{C_{3}}\left(D_{3, t}\right)^{2}-\frac{1}{C_{4}}\left(D_{4, t}\right)^{2}=0
$$

Note that these last two conditions state the effective collaborative rules for the enterprises belonging to a CO-DESNET. In practice, the agreement to be a network partner states that each firm agrees in producing, for the network needs, by using its own production capacity at a rate which must be balanced with the capacities of the other network partners: no enterprise will receive a demand for products "unbalanced" with respect to the others. In addition, conditions (12) to (14) will impose an equilibrium on the network internal prices.

The global management problem results from the whole set of conditions (1) to (16) above stated. It consists of a large-scale non-linear optimization problem: existence of an optimal solution can be proven according to standard optimisation theory results (Brandimarte \& Villa, 1995).

By applying Lagrangian relaxation, the complete optimisation problem can be split into four optimization sub-problems, all interrelated together, each one linked to a component firm $n$, namely:

- for the firm $n=1$

$$
\begin{aligned}
& \min _{X, P, Y} \Pi_{1}=\sum_{t}\left(-P_{1, t} X_{n, t}+c_{1} I_{1, t, F}+r_{1} Y_{1, t}+\right. \\
& \left.+\alpha_{t} X_{1, t}+\gamma_{t} \cdot \frac{1}{C_{1}}\left[X_{1, t}\right]^{2}+\left(v_{t}-\delta_{t}\right) P_{1, t}\right)
\end{aligned}
$$

- for the firm $n=2$

$$
\begin{aligned}
& \min _{X, P, Y} \Pi_{2}=\sum_{t}\left(-P_{2, t} X_{2, t}+c_{2} I_{2, t, F}+r_{2} Y_{2, t}+\right. \\
& \left.+\alpha_{t} X_{2, t}-\gamma_{t} \frac{1}{C_{2}}\left[X_{2, t}\right]^{2}-v_{t} P_{2, t}\right)
\end{aligned}
$$

- for the firm $n=3$

$$
\begin{aligned}
& \min _{Q, Z, W, D} \Pi_{3}=\sum_{t}\left(Q_{3, t} Z_{3, t}+r_{3} W_{3, t}+c_{3, M} I_{3, t, M}+\right. \\
& \quad+c_{3, P} I_{3, t, P}-S_{t} D_{3, t}-\alpha_{t} Z_{3, t} \\
& \left.-\eta_{t} \frac{1}{C_{3}}\left[\mathrm{D}_{3, t}\right]^{2}+\beta_{t} Q_{3, t}+\omega_{t} D_{3, t}\right)
\end{aligned}
$$

- for the firm $n=4$ 


$$
\begin{aligned}
& \min _{Q, Z, W, D} \Pi_{4}=\sum_{t}\left(Q_{4, t} Z_{4, t}+r_{4} W_{4, t}+c_{4, M} I_{4, t, M}+\right. \\
& +c_{4, P} I_{4, t, P}-S_{t} D_{4, t}-\alpha_{t} Z_{4, t} \\
& +\eta_{t} \frac{1}{C_{4}}\left[\mathrm{D}_{4, \mathrm{t}}\right]^{2}+\left(\delta_{t}-\beta_{t}\right) Q_{4, t}+ \\
& \left.+\omega_{t} D_{4, t}\right)
\end{aligned}
$$

In above stated formulations, the newly introduced variables (in Greek letters) denote the Lagrangian variables, by which the constraints have been included into the augmented global functional to be minimized. Maximization with respect to Lagrangian variables will allow to obtain the co-ordination conditions.

\section{COOPERATION CONDITIONS AMONG PARTNER FIRMS}

Conditions which assure collaborative operations of the firms belonging to a $D E S N E T$, can be obtained by analysing the Lagrangian variables, introduced in the relaxed formulation, since Lagrangian variables denote co-ordination costs. Some particularly interesting examples of such conditions are outlined in the following.

(a) The variable $\alpha_{t}$ denotes the co-ordination cost concerning the volumes exchanged between the two suppliers and the two buyers, as stated by constraint (10). In case the offered volume is greater than the requested demand, such cost is greater than zero. Then, from (17) and (18), both first-stage producers are pushed to reduce their throughput. For the second-stage buyers, from (19)(20), the effect is opposite.

(b) The variable $\gamma_{t}$ refers to the co-ordination cost concerning volumes of production to be shared between the two first-stage suppliers, related to condition (15). The squared valued functions in (17) and (18) force a split of production volumes respectively depending on the rate between the production throughput $X_{n, t}$ and the firm capacity utilization, estimated by $\frac{1}{U_{n, t}}=\frac{X_{n, t}}{C_{n}}$, for $n=1,2$.

Then, cost $\gamma_{t}$ forces to minimize unbalancing between concurrent producers.

The Lagragian variable $\eta_{t}$ operates in a similar way as $\gamma_{t}$, on the two firms belonging to the second stage, as in constraint (16).

(c) The variable $v_{t}$ represents an "horizontal co-ordination" cost concerning a potential difference between prices offered by the concurrent first-stage suppliers, as referred to constraint (12). In case such cost will increase, the first supplier $(n=1)$ is compelled to pay an adjoint quantity of production for each unit sold by the second supplier $(n=2)$, as shown in (17) and (18). A similar effect is due to the second-stage "horizontal co-ordination" cost $\beta_{l}$, as in (19)(20), now associated to constraint (14). This effect is a demonstration of how a CO-DESNET should operate in order to regulate the financial transactions between concurrent producers: prices must be maintained as equal as possible otherwise a "dumping" attempt will be paid in terms of reduction of the 
planned throughput.

(d) Again referring to price management within the network, the variable $\delta_{t}$ is referred to the "vertical co-ordination" between the price proposed by upstream producers $(n=1,2)$ and the price which downstream buyers $(n=3,4)$ could agree to pay, i.e. associated to constraint (13). As soon as $\delta_{t}$ decreases, buyer 3 should pay an additional cost per part to supplier 1. The pricecoordinating costs are strictly interrelated together. Any price variation between two firms of the same stage should immediately imply price unbalancing on the other stage and between the firms belonging to the other stage, as well. This effect is evidenced by the Lagrangian variables associated to the "triangular conditions" (12-(14).

(e) The variable $\omega_{t}$ is the co-ordination cost associated to the equilibrium requirement between volumes offered by downstream firms $(n=3,4)$ and demands of the end customer in (11), the real exogenous input of the whole DESNET. The sensitivity of the network management to $\omega_{t}$ can be read as a cost paid by end products' producers to the end customer if the volumes offered are greater than the demand (i.e., a discount to push sales).

\section{FINAL CONSIDERATIONS}

The performance evaluation of the DESNET above presented has to be completed by better specifying profitability conditions. In practice, a potential manager of a network of this type should be able to prove that the required investment for the network organization should be so advantageous as to promote a real collaboration among partners (Bullinger et al., 2002; and Supply Chain Council, 2001).

It should be approached, indeed, the problem of validating the economic advantage of an investment devoted to promoting a DESNET, by investigating if there is clear interest to increase as much as possible its dimension, as it could be useful from the structural point of view of acquiring even larger production capacity and wider market share. An answer is that complexity-induced costs cannot overcome advantages offered by the "protected market", advantages consisting of the reduced global transaction costs. Once the most relevant transactions costs are recognized as those due to the procurement of information about the market, the monitoring of the trade partners performance, the addressing of problems that might arise in trade relationships and the risk of partners opportunistic behaviour ( V.Grover, M.K. Malhotra, 2003), then enterprises can be convinced of the advantage to stipulate multi-agent contracts, by specifying the contribution of each partner and the respective rate of the global income. This is the first suggestion that the presented network model gives in terms of "equilibrium-assuring management".

A further problem concerns how to evaluate the investment for the development and innovation of a DESNET. Several classic criteria have been introduced to perform an analysis of the return of investment, generally based on the balancing between costs and return flows (Rossetto \& Villa, 2003). Recently, a method based on the concept of "real options" (i.e. by noting that, when an investment is decided, the decisionmaker still has the opportunity to choose at which time to invest as well as in which 
type of realization) became more and more interesting. Referring to a DESNET, this method seems to be even interesting, because it allows a supply chain organizer to analyse alternatives: if to enlarge the chain, or to improve the connections among agents, or to promote specific technologies at some partner. Obviously, a robust estimation of the return of investment, at any option time and for any potential option, is mandatory. The model proposed in the previous Sections can be used as a "planner" of the network operations over a future time horizon: thus, it can be applied as an "observer" of the network evolution in front of modification hypotheses, and an "estimator" of the network innovations' advantage.

Acknowledgements: This research has been partially supported by the Italian Ministry of Education, University and Research, within a national-interest research programme PRIN.

\section{REFERENCES}

Brandimarte, P., and Villa, A. (1995). Advanced Models for Manufacturing Systems Management, CRC Press, Boca Raton.

Bullinger, H-J., Kuhner, M., and Van Hoof, A. (2002). Analyzing supply chain performance using a balanced measurement method, Int. Journal of Production Research, 40, 3533-3543.

Faratin P., Sierra C., Jennings, Nick R., (1998) Negotiation decision functions for autonomous agents, Robotics and Autonomous Systems, 24, 159-182

Grover, V., Malhotra, M.K. (2003), Transaction Cost Framework in operations and supply chain management research: theory and measurement, Journal of Operation Management, 21, 457-473.

Huang, C-Y., and Nof, S. Y. (2000). Autonomy and viability - measures for agent-based manufacturing systems, Int. Journal of Production Research, 33, 4129-4148.

Rossetto, S., and Villa, A. (2003). Evaluating capital investment for worldwide supply chain organization, in Proc. IFAC Int. Workshop on Intelligent Assembly and Disassembly, T. Borangiu (ed), Bucharest, pp. 215-220.

Sethi, S.P., and Zhang, Q. (1994). Hierarchical Decision Making in Stochastic Manufacturing Systems, Birkhauser, Boston.

Supply Chain Council (2001). Supply Chain Operations Reference Model, see the web site: http://www supply-chain.org.

Simchi-Levi D., Kaminski P., Simchi-Levi E., (2000) Desining and managing the Supply Chain - Concepts Strategies and Cases Studies, McGraw-Hill.

Villa, A. (2001). "Introducing some supply chain management problems", Int. J. Production Economics, $73,1-4$

Villa, A. (2002). "Emerging trends in large-scale supply chain management", Int. Journal of Production Research, 40, 3487-3498.

Wellman, M.P. (1993), Market Oriented Programming Environments and its Applications to Multicommodity Flow Problems, Journal of Artificial Intelligence.

Yung S.K., Yang, C.C., (1999) A new approach to solve supply chain management problems by integrating multy agent technology and constraint networks, Proceedings of the $32^{\text {nd }}$ Hawaii International Conference on System Science.

Zheng, L., and Possel-Dolken, F. (2002), Strategic Production Networks, Springer-Verlag, Berlin. 